\title{
Juventudes e interseccionalidad: Proyectos ocupacionales de estudiantes del nivel medio de la ciudad de Rafaela
} Youth and Intersectionality: Occupational Projects of Middle School Students in Rafaela City

\author{
Cecilia Dionisio y Hugo Lewin ${ }^{1}$
}

\begin{abstract}
Resumen
Este artículo presenta los resultados preliminares del proyecto de investigación «FUTURO (IM)PERFECTO. Percepciones de las/os estudiantes del último año del nivel medio sobre las alternativas profesionales y la formación universitaria pública en la ciudad de Rafaela», actualmente en curso en la Universidad Nacional de Rafaela. El objetivo principal fue indagar el grado de definición de los «Proyectos Profesionales» de estudiantes próximos a egresar de establecimientos de enseñanza media de Rafaela considerando las variables de género, clase social y modalidad educativa. Se adoptó una metodología cualitativa, utilizando grupos focales y entrevistas en profundidad que evidenciaran los nexos entre los sujetos y sus contextos. Para el análisis, se consideró la perspectiva interseccional entendiendo que las normativas de género son dependientes de las intersecciones con modalidades raciales, de clase, étnicas, sexuales o regionales. Se encontró que las prefiguraciones ocupacionales de las/os entrevistadas/os están atravesadas por estereotipos y mandatos de género en consonancia con el modelo patriarcal. A la vez, el nivel socio-económico condicionó el acceso a los estudios superiores y a las ocupaciones prefiguradas. Los sectores sociales más empobrecidos evidenciaron que el tiempo es un recurso central para pensarse en el ámbito laboral y los estudios superiores. Finalmente, encontramos que las mujeres provenientes de sectores sociales empobrecidos eran el grupo que atravesaba la mayor cantidad de inequidades, en donde las variables género y nivel socio económico se combinaban para restringir sus posibilidades en los estudios superiores y el mercado laboral, dejándolas en una situación de mayor vulnerabilidad social.
\end{abstract}

\section{Palabras clave}

Juventudes, género, proyecto ocupacional.

\section{Abstract}

This article presents the preliminary results of the research project: «(IM)PERFECT FUTURE. Perceptions of students of the last year of middle school on professional alternatives and public university education in the city of Rafaela», currently in progress at the National University of Rafaela. The main objective was to investigate the degree of definition of the «Professional Projects» of students close to graduating from secondary schools in Rafaela considering the variables of gender, social class and educational modality. A qualitative methodology was adopted, using focus groups and in-depth interviews that showed the links between the subjects and their contexts. For the analysis, the intersectional perspective was considered with the understanding that gender norms are dependent on intersections with racial, class, ethnic, sexual or regional modalities. It was found that the occupational prefigurations of the interviewees are crossed by stereotypes and gender mandates in line with the patriarchal model. At the same time, the socioeconomic level conditioned access to higher education and prefigured occupations. The most impoverished social sectors showed that time is a central resource to think about work and higher education. Finally, we found that women from impoverished social sectors were the group that suffered the most inequities, where the variables of gender and socioeconomic level were combined to restrict their possibilities in higher education and the labour market, leaving them in a situation of greater social vulnerability.

\section{Keywords}

Youth, gender, occupational projects.

\section{Cómo citar/Citation}

Dionisio, Cecilia; Lewin, Hugo (2020). Juventudes e interseccionalidad: proyectos ocupacionales de estudiantes del nivel medio de la ciudad de Rafaela. Revista de Sociología de la Educación-RASE, 13 (3), 415-431. http://dx.doi.org/10.7203/RASE.13.3.16428. 


\section{Introducción}

Desde la mirada de la sociología de la educación se observa que, en la actualidad y desde hace unas décadas, los procesos de inserción al mundo laboral se configuran como transiciones prolongadas hacia destinos inciertos (Roberts, 1997). En el marco de la crisis de la «sociedad salarial», tal como plantea Castel (1997), se reduce drásticamente el trabajo asalariado estable, se incrementa la rotación en el empleo a lo largo de la vida, se acrecienta el trabajo no registrado y el desempleo. Asimismo, la tercerización de la economía, los procesos de des-industrialización y el mayor peso de la economía de servicios han diversificado la demanda laboral. En este contexto, sostiene Sennet (2000), se transforman las transiciones juveniles a la adultez, tornándose no lineales. Mientras que en el período de postguerras la transición entre el mundo escolar y laboral se encaminaba sin desvíos o demoras hacia la toma de roles sociales adultos, desde la última década del siglo xx la transición se despliega a través de caminos diversos, cada vez más heterogéneos e individualizados (Miranda, 2007; Machado País, 2004; Casal, 2000). En este marco, cobra mayor relevancia la capacidad de los individuos para gestionar sus propias biografías (White y Wyn, 2008).

En Argentina, (Miranda, 2015; Bendit, y Miranda, 2013; Corica, 2012; Filmus et al., 2001) analizan el papel de la escuela secundaria en el tránsito hacia la adquisición de competencias para la inserción laboral y la asunción de responsabilidades típicamente asociadas a la adultez. Por una parte, en las sociedades latinoamericanas, la educación secundaria se convirtió cada vez más en el umbral mínimo necesario para garantizar una integración social plena (Filmus, 2015). Así, se sancionó, en 2006, la Ley de Educación Nacional (N. ${ }^{\circ}$ 26206) que estableció la obligatoriedad de la escuela secundaria en Argentina. Esta legislación garantizó el acceso a la educación de nivel medio a jóvenes de entre trece y dieciocho años, provenientes de sectores sociales que nunca antes lo habían tenido. Como resultado de esta política, entre 2006 y 2018, la matrícula del nivel secundario aumentó un 11\% y la cantidad de jóvenes graduados creció un 39\% (Cardini y D'Alessandre, 2019). Sin embargo, persisten una serie de diferenciaciones en el interior del sistema que conllevan experiencias educativas desiguales, evidenciándose que los sectores de menores recursos económicos, las poblaciones rurales y particularmente las mujeres que tienen a su cargo las tareas domésticas, aún enfrentan serias dificultades para culminar la escuela secundaria (Filmus, 2015).

A su vez, la escuela media es cuestionada en múltiples aspectos, tal como plantea (Miranda, 2013). En primer lugar, por su desvinculación con la formación en las capacidades que demanda el trabajo y por no garantizar la inserción en el mercado laboral en condiciones más ventajosas. También se cuestiona su capacidad para formar en valores propios de la ciudadanía, en un contexto de globalización y consumo, que en los países latinoamericanos han producido exclusión, desafiliación y marginación de los consumos materiales y simbólicos. Por último, la escuela media es cuestionada en su capacidad para formar en los conocimientos básicos que demandan los estudios superiores. En relación a este último punto, la educación superior ${ }^{2}$ también se encuentra con el desafío de generar políticas de integración destinadas a aquellos sectores que anteriormente no accedían a este nivel educativo.

Así, el desempeño de las/os jóvenes de distintos sectores frente al desafío de la inserción y la permanencia en los puestos de trabajo se relaciona con sus capitales sociales, culturales y educacionales. La estructura del capital (Bourdieu, 2001) acerca probabilísticamente determinadas oportunidades y excluye otras. Es sabido que las trayectorias menos lineales, de mayor trashumancia, junto a las chances de des-

2 Argentina cuenta con 56 universidades nacionales. Éstas son gratuitas y requieren la finalización de la escuela secundaria para acceder a ella, a excepción de aquellos sujetos mayores de 25 años que acrediten experiencia específica en el ámbito disciplinar al que quieran incorporarse. 
ocupación, son más frecuentes entre las/os jóvenes que pertenecen a grupos sociales desfavorecidos en materia económica y educativa. En tanto que la experiencia escolar de los sectores socioeconómicos altos es intensa y construye una subjetividad «pro aprendizaje», la experiencia escolar en los sectores de menores recursos suele ser más exigua (Duschastzky y Corea 2000). Se suma a esta desigualdad, la menor disponibilidad de moratoria social (Margulis, 1996) en estos últimos, por lo que sus posibilidades de ensayo y error y de apuesta a la educación los colocan en posiciones desfavorables para competir por puestos que son escasos.

En este marco de desigualdades, incertidumbre, riesgo y dificultades para prefigurar tránsitos medianamente previsibles hacia las crecientes responsabilidades de la vida, las transiciones entre el mundo escolar y laboral hoy en día son más diversas y heterogéneas. No obstante, la mayor parte de las investigaciones en este campo se realizan en regiones urbanas de gran concentración, por lo que investigar en ciudades más pequeñas y poco analizadas aporta a la construcción de conocimientos situados que contemplen la realidad de otros territorios. Es así que se planteó la investigación «FUTURO (IM)PERFECTO. Percepciones de las y los estudiantes del último año del nivel medio sobre las alternativas profesionales y la formación universitaria pública en la ciudad de Rafaela», actualmente en curso en la Universidad Nacional de Rafaela (UNRaf). Su objetivo principal fue indagar en el grado de definición de los «Proyectos Profesionales» de las/os estudiantes próximos a egresar de los establecimientos de enseñanza media de Rafaela, considerando las variables de género, clase social y modalidad educativa de la escuela. Este artículo presenta los resultados preliminares de la investigación, asumiendo que conocer las autopercepciones del futuro y las expectativas de futura inserción de las/os jóvenes permitirá delinear estrategias en el campo universitario para una mejor llegada a ese nivel de formación.

\section{Ocupaciones juveniles desde la interseccionalidad}

El género, en tanto construcción cultural, social y simbólica, se relaciona estrechamente con las posibilidades de prefigurarse una ocupación, puesto que la cultura delimita las tareas habilitadas y legítimas para cada uno de los géneros. Como plantea Scott (1999), el género es una forma primaria de relaciones significantes de poder que establece el marco de referencia que estructura la percepción y la organización de toda la vida social, incluyendo la distribución y el acceso a los recursos materiales y simbólicos. Así, se construye un esquema clasificatorio binario que establece un «orden» basado en la condición de género a partir del cual se determinan expectativas y atributos «esperables»: la «normatividad genérica». Desde allí, se socializa a los sujetos para que cumplan con los mandatos de género que prescriben los comportamientos, expectativas, roles y espacios asignados, así como las emociones permitidas y las sensaciones prohibidas (Subirats y Brullet, 1988). Sin embargo, el género no siempre se establece de manera coherente o consistente en contextos históricos diferentes, por lo que resulta imposible desligarlo de las intersecciones políticas y culturales en que se produce y mantiene (Butler, 2001). Así, el concepto de interseccionalidad, acuñado originalmente por Crenshaw (1989), se propone como una herramienta para analizar las características que asumen las normativas de género en el entrecruce con el contexto histórico y las modalidades raciales, de clase, étnicas, sexuales o regionales.

En el campo de la educación y el trabajo, diversas investigaciones (García Ramírez, 2016; Panaia, 2013; Eurydice, 2010; Arango Gaviria, 2006, Subirats, 1999) afirman que las mujeres suelen optar por carreras relacionadas con las humanidades, educación y ciencias de la salud; mientras que los varones eligen carreras dentro del abanico de las «ciencias duras», como las ingenierías. Estas elecciones no son casuales 
y se relacionan con el modelo que la cultura patriarcal ha promovido, en el cual se asocia la afectividad -«el corazón»- con las mujeres, a la vez que el espacio privado/doméstico; mientras que la inteligencia - «la razón»- se asocia con los hombres al mismo tiempo que el espacio público. En consecuencia, los estereotipos de género condicionan las trayectorias académicas y profesionales futuras, repercutiendo, a largo plazo, en la segregación horizontal y vertical que se da en el mercado de trabajo y que es fuente de las desigualdades de género (González Lopez, 2009). Tal como plantea Fraser (1997), el género estructura la división del trabajo remunerado ya que las ocupaciones industriales y profesionales mejor pagas son ocupadas por hombres, mientras que las ocupaciones «de cuello rosa» y de servicio doméstico, mal pagas, son ocupadas por mujeres.

Conjuntamente con una brecha salarial de género que se comprueba mundialmente (según datos de la OIT las mujeres ganan alrededor de un $25 \%$ menos que los hombres), las mujeres tienen la mayor carga respecto del trabajo doméstico. En Argentina, las mujeres destinan casi el doble de horas a las tareas del hogar que los hombres produciendo una estructura política-económica que explota, margina y priva según el género (D’alessandro, 2019). En ese sentido, la incorporación progresiva de las mujeres al mundo del trabajo implicó una sobreexplotación de sí mismas, a la vez que las enfrentó a una serie de obstáculos tales como los «pisos pegajosos» y los «techos de cristal».

En este sentido, la economía feminista, desde una perspectiva interseccional, ha construido el concepto de «feminización de la pobreza» para dar cuenta de que la pobreza se encuentra íntimamente relacionada con la desigualdad de género y se retroalimentan. Por una parte, la falta de acceso y las desigualdades de género frente a la educación, producen un efecto multiplicador con consecuencias negativas, ya que repercute en empleos de mala calidad y baja remuneración (Baeza Leiva, 2015). En consecuencia, las mujeres suelen tener peores cualificaciones laborales, presentan mayores dificultades para conseguir trabajo y representan la mayor parte de la población en trabajos precarizados. Por otra parte, la carga del trabajo doméstico produce una «pobreza de tiempo» en donde las mujeres de menores recursos se encuentran forzadas a cumplir una doble jornada laboral que produce peores condiciones en la calidad de vida, repercutiendo inclusive en salud (Esquivel, 2016).

Otro componente clave en este análisis, es el la frecuente estigmatización de las juventudes pertenecientes a sectores populares. D’alessandro (2019) retoma la expresión «ni-ni» que se utiliza en Argentina para referirse a las/os jóvenes que ni estudian ni trabajan. La autora resalta que, además de la fuerte carga estigmatizante que esta denominación conlleva, suele omitir el hecho de que el 63\% de esas/os jóvenes «ni-ni» son mujeres que trabajan en sus casas, cuidando a sus hijas/os, hermanas/os menores o familiares enfermas/os. Así, el carácter invisible del trabajo doméstico impacta mayormente en las mujeres y de manera más significativa en aquellas de origen social más desfavorecido. En este sentido, las situaciones de desventaja que enfrentan las mujeres jóvenes y otras identidades feminizadas, se agudizan cuando provienen de sectores desfavorecidos tanto económica como simbólicamente, puesto que combinan las desigualdades económicas provocadas por el propio funcionamiento del sistema capitalista con las desigualdades simbólicas que se construyen socialmente (Calderon y Campos Rios, 2013).

\section{Metodología}

Se adoptó la metodología cualitativa de investigación, lo que significa trabajar con una perspectiva comprensivista. Procuramos describir e interpretar el sentido que para los actores tienen sus prácticas, des- 
plegando dos técnicas de conversación en un conjunto definido de casos: grupos focales y entrevistas en profundidad. A partir de las experiencias narradas de manera biográfica fue posible reconstruir las tramas significativas que evidencian los nexos entre cada sujeto y su contexto (Guevara y Belleli, 2015).

La población de estudio se conformó por estudiantes próximos a egresar de los establecimientos de enseñanza media de Rafaela. De ese universo, seleccionamos las tres modalidades educativas que juntas agregan a la mayoría de la población estudiantil: educación artística, educación técnico profesional y educación permanente de jóvenes y adultos. Quedaron afuera de esta selección la educación especial, la desarrollada en contextos de encierro, la rural, la intercultural bilingüe y la domiciliaria y hospitalaria. Este recorte se debió a cuestiones de factibilidad derivadas de la accesibilidad y los plazos de ejecución del proyecto. De este modo, la muestra quedó conformada por estudiantes próximos a egresar de los establecimientos de enseñanza media de la ciudad de Rafaela, cuya modalidad educativa fuera educación artística, educación técnico profesional o educación permanente.

Asimismo, se consideraron las variables de género y nivel socio-económico para el diseño de la muestra. Para definir la variable nivel socio-económico, se realizó una indagación exploratoria con directivos y docentes de las escuelas de la ciudad, acerca de los sectores sociales que asistían a cada escuela. En términos generales, la clasificación de las/os estudiantes que realizaron se basó en su poder adquisitivo y capacidad de consumo, clasificándolos como «medios» o «bajos/pobres/empobrecidos». Se realizó entonces, una división conceptual de la población en tres sectores alto/medio/bajo. Luego, se aplicó a las/os participantes el Cuestionario Simplificado de Nivel Socio-Económico (2015) de la Sociedad Argentina de Investigadores de Marketing y Opinión, para sustentar de manera más acabada la pertenencia a alguno de esos sectores. Por su parte, la variable género se definió a partir de la Ley de Identidad de Género (26 743) como la vivencia interna e individual del género tal como cada persona la siente. Los sujetos entrevistados se autopercibieron como mujeres o varones, por lo que retomamos esa clasificación.

Con el propósito de validar la guía de conversación utilizada en los grupos focales, se realizaron seis entrevistas en profundidad, que no consideraron la variable modalidad de educación de la escuela. Luego, se realizaron las entrevistas con directivos de escuelas, donde acordamos una agenda para la realización de los grupos focales y seleccionamos a las/os participantes contemplando el entrecruce de variables. Cabe destacar que, las escuelas que nos recibieron se caracterizaron por ser estatales y agrupar a estudiantes provenientes sectores económicos medios y bajos. Las escuelas a las que asisten poblaciones de niveles socio-económico altos en Rafaela son de carácter privado y no permitieron el ingreso a sus establecimientos, por lo que no fue posible abordar ese sector. Así, se realizaron nueve grupos focales, integrados por entre seis y ocho participantes cada uno, en un total de cinco escuelas. La duración de cada grupo focal fue de entre 50' y 1h 10' cada uno y su coordinación fue compartida por dos o tres integrantes del equipo de investigación. Considerando que solamente existe una escuela de modalidad educativa artística en la ciudad, los grupos focales se distribuyeron en función de las variables tal como se muestra en la Tabla I, donde SM refiere a sector social medio y SB a sector social bajo: 


\section{Tabla I. Distribución de la cantidad de grupos focales realizados en función de las variables en estudio}

\begin{tabular}{ccccc}
\hline \multirow{2}{*}{ MODALIDAD } & VARONESES & & MUJERES \\
\cline { 2 - 5 } & SM & SB & SM & SB \\
\hline Técnica & 1 & 0 & 0 & 1 \\
\hline Permanente & 1 & 1 & 2 & 1 \\
\hline Artística & 1 & 0 & 1 & 0 \\
\hline
\end{tabular}

Fuente: elaboración propia.

\section{Resultados preliminares}

\subsection{Ocupaciones generizadas}

La indagación acerca de las ocupaciones prefiguradas por las/os entrevistadas/os se estableció en torno tres ejes: las elecciones personales, las ocupaciones de sus madres/padres, y las habilidades/ capacidades necesarias para desempeñar una ocupación determinada. En todos los ejes observamos la preeminencia del modelo patriarcal y heteronormativo que clasifica y jerarquiza las ocupaciones en función del género.

Las ocupaciones prefiguradas por entrevistadas mostraron una relación estrecha con el sector de servicios (principalmente limpieza y producción de alimentos), las ciencias humanas (psicología, psicopedagogía, sociología), el ámbito de la docencia (profesorados de nivel inicial, primario y secundario) y otras relacionadas a las ciencias médicas pero dentro de la rama de los «cuidados» (obstetricia, veterinaria de animales pequeños, pediatría, biología marina). Por su parte, en los varones se encontraron ocupaciones relacionadas a ingeniería, abogacía, arquitectura y profesorados de educación física, geografía e historia. Además, se les preguntó a las/os entrevistadas/os si consideraban que existían ocupaciones típicamente de mujeres o varones. En principio, la mayor parte de los grupos respondía que no, pero luego comenzaban a mencionar un amplio abanico de ocupaciones asociadas de modo estereotipado al género. Desde allí, se indagó acerca de los «atributos» que hacían de esas ocupaciones «trabajos de mujer» o «trabajos de varón». Las mujeres fueron rápidamente asociadas a atributos como «detallista», «prolijidad», «paciencia», «delicadeza» y «agilidad» para tratar con personas; mientras que «fuerza», «autoridad» y «ser bruto» se asoció a los varones. Estos estereotipos de género llevan a asignar a las mujeres lo que Hochschild (1983) llamó «trabajo emocional», intentando dar cuenta de la carga emocional/afectiva que conllevan. A la vez, las ocupaciones feminizadas suelen estar más precarizadas y suelen ser menos prestigiosas (Pozzio, 2012).

Se analizó, también, la relación existente entre modalidades de las escuelas y elecciones ocupaciones. En las modalidades educación permanente y educación artística no se evidenciaron nexos demasiado directos ni estables entre las ocupaciones prefiguradas y los aprendizajes recibidos durante el secundario. No obstante, en las escuelas de educación técnica se marcó una diferencia significativa: mientras que los varones continuarían estudiando o trabajando en ámbitos relacionados a las ingenierías, metalurgia, informática, talleres de carpintería e incluso trabajos en empresas de energía; ninguna de las mujeres mostró interés por esas ocupaciones. Además, la mayoría de las mujeres cursaba dentro de la orientación «programación» y mencionaron que la orientación «metalurgia» era «de hombres». Por esa razón, ellas no habían elegido esa orientación dentro de la escuela y recordaban compañeras de esa rama que no «habían quedado» en la empresa metalúrgica donde habían hecho las pasantías, 
a diferencia de sus pares varones. A la vez, emergieron una serie de prácticas docentes a través de las cuales la escuela encarna y reproduce normas hegemónicas de género.

\section{Moderadora: ¿Y acá en escuela perciben eso? ¿Diferencias entre varones y mujeres? Participante 1: Por abi, a veces dicen: «necesito 2 chicos que vayan a hacer fuerza». \\ Participante 2: Los talleres de electrónica los daban todos hombres. \\ (Mujeres, educación técnica, sector bajo).}

De esta manera, la escuela prescribe tareas y lugares socialmente permitidos en función del género. El aprendizaje de estas reglas y normas involucra un disciplinamiento constante de todas las partes del cuerpo del sujeto, haciéndose más eficaz cuanto más tácito permanece. Así, lo «masculino» y lo «femenino» se define relacionalmente como categorías opuestas y complementarias que se codifican en los modos de mantener el cuerpo y comportarse (Bourdieu, 2000). A partir de allí, la discusión sobre la división del trabajo doméstico ha tomado una relevancia central para la observar las inequidades de género. En los grupos focales realizados encontramos por un lado, que la mayor carga del trabajo doméstico y de cuidados recae sobre las mujeres; pero también que algunas tareas domésticas aparecen generizadas. P2: Yo vivía con mi abuelo que siempre fue así como un machista extremo, ¿no? que era que «el
nene le ayudaba a hacer el asado», jamás me dejó acercarme a la parrilla... me da una bronca...
porque a mi me encanta cocinary todo eso, jamás me dejó acercarme a la parrilla. Y era «a tal hora
todas las nenas tenían que estar ayudando en la cocina a hacer el puré de papas y poner la mesa»...
(Mujer, educación permanente, sector bajo).

P4: En mi casa tengo una hermana, más chica que yo y cuando quiero limpiar algo de la casa, viene mi mamá y me dice: «dejá que lo hace tu hermana». Yo le pregunto «ippor qué?», y me responde: «porque lo bace mejor».

(Varón, educación permanente, sector medio).

Estos actos de autoridad legitiman construcciones de género que son producto de un trabajo continuado y, por tanto, histórico. Así, el sistema patriarcal coloca a las mujeres en un estado permanente de inseguridad corporal que es, en última instancia, un estado de dependencia simbólica (Bourdieu, 2000). En este sentido, también observamos que a las mujeres se les presentaba como un hecho altamente conflictivo el estudiar fuera de su ciudad de origen por diferentes situaciones ligadas al miedo. Los miedos, tanto de sus familiares como propios, se relacionaban a distintas imágenes internalizadas de peligros y riesgos que circulan en la discursividad social, pero que están vinculadas estrechamente a la condición de ser mujer en tanto «ser mujer conlleva un miedo específico, diferencial y añadido a la representación subjetiva del miedo o la inseguridad que puede sufrir un hombre» (Añover López, 2012: 29).

M: Ah, iy te vas a vivir a Santa Fe?

P3: Si, lo más probable, si es que no me frena nada.

M: ¿Cómo? 


\section{P 3: Si es que mi papá no me frena nada, si no se arrepiente.}

P4: No, a mi no me dejan irme, no me quieren despegar de ellos. No quieren, o sea, ni ganas de que me vaya.

M: ¿Y por qué les parecen que no se animan?

P1: Porque pasamos a ser independientes de ellos.

P2: Por todo lo que está pasando y... como que se te va la nena de la casa también. Ya no está bajo tu cuidado.

P1: Aparte existe como un pensamiento tipo: «es tu bija mujer y se va».

(Mujeres, educación permanente, sector medio).

Además, estudiar fuera de Rafaela implica generalmente mudarse a alguna de las ciudades que combinan la mejor relación entre oferta académica y cercanía geográfica: Santa Fe, Rosario o Córdoba. Estas ciudades se configuran como grandes centros urbanos que suelen asociarse a espacios más amenazantes. Desde la mirada del urbanismo feminista, Falú (2014) afirma que los espacios públicos de las ciudades siguen siendo mayoritariamente masculinos y que uno de los factores que dificultan la apropiación de los territorios por parte de las mujeres son las violencias que se ejercen contra éstas en los espacios públicos. Estas violencias se configuran como un mecanismo político cuyo fin es mantener a las mujeres en desventaja y desigualdad en el mundo y en las relaciones con los hombres, excluyéndolas del acceso a bienes, recursos y oportunidades. Así, las mujeres carecen de autonomía a la hora de desplazarse por la metrópoli, lo cual contribuye a desvalorizar, denigrar y amedrentarlas, a la vez que reproduce el dominio patriarcal a través del miedo como factor intergeneracional (Lagarde, 2006). El miedo, tan presente en los relatos de las entrevistadas, está absolutamente ausente en los entrevistados, para quienes irse a estudiar a otra ciudad se asocia a la promesa de libertad y la autonomía.

\section{P3: Para probar algo nuevo. Después además me va a servir un montón porque siempre estoy aprendiendo. Asi que voy a cocinarme yo, voy a elegir yo qué comer y voy a ver yo qué hacer. \\ $M:$ ¿Y sus padres qué dicen al respecto? \\ P1: Y siempre que se puede, si se puede, no te van a decir que no. Eso supongo yo. \\ P3: Claro, no creo que les guste la idea de que te vayas tan lejos, pero... \\ P1: Respetan tu decisión. Por lo general.}

(Varones, educación técnica, sector medio).

\subsection{Las elecciones de los sectores medios}

Los grupos focales mostraron, también, que el nivel socio-económico marcaba diferencias importantes en la posibilidad de prefigurarse una ocupación a futuro. Para poder contextualizar esos hallazgos, debe considerarse que en Rafaela existen cuatro instituciones de educación superior de carácter pública y, por tanto, gratuitas (dos universidades nacionales, una extensión áulica de otra universidad nacional y un instituto terciario provincial) y cuatro instituciones de educación superior privada y, por tanto, pagas (dos universidades privadas y dos extensiones áulica de otras universidades privadas). A la vez, la oferta académica de las instituciones públicas contempla carreras diferentes de aquellas que se ofrecen en las instituciones privadas. De este modo, existe un amplio abanico de carreras universitarias a las que las/os habitantes de Rafaela solo pueden acceder pagando una cuota o mudándose a otra ciudad para estudiarlas. 
La mayor parte de las/os entrevistadas/os de sectores sociales medios manifestó intenciones de continuar estudios superiores en el instituto terciario (que reúne la mayoría de los estudios de profesorados) o en universidades públicas o privadas, tanto en Rafaela como en otras ciudades. Los estudios superiores fueron considerados como un requisito necesario para conseguir trabajo lo cual se marca como una diferencia en relación a otras generaciones:

P2: «Antes salias de la secundaria y ya tenías trabajo o conseguías y abora si no tenés título universitario o algo terciario, no conseguís trabajo».

(Mujer, educación permanente, sector medio).

Sin embargo, «conseguir trabajo» no implica realizar cualquier tarea. El título universitario representa para estos sectores una posibilidad de ascenso social y la oportunidad para conseguir «mejores trabajos». Esta mejoría se relaciona, principalmente, con ocupar posiciones de jerarquía en el ámbito laboral que, a la vez, se diferencian de otros tipos de ocupaciones consideradas «feas» o «aburridas». El «gusto» por la ocupación realizada aparece entonces como una marca de privilegio a la hora de poder elegir que estudiar, ya que «estudiar algo que te guste» implica contar con el tiempo y el dinero para poder hacerlo:

\footnotetext{
P3: «No levantarme cada mañana y estar en una fábrica ocho horas. En cambio, me gustaría levantarme para hacer las ocho horas haciendo algo que a mi me gusta».

(Varón, educación artística, sector medio).
}

P5: Obviamente que si estudiás o trabajás en un ambiente que te gusta o te recibís de la carrera que te gusta, no te va a molestar tanto trabajar en el mismo lugar por muchos años o por mucho tiempo, en cambio si estás en un trabajo en el que quedaste por quedar o porque es una de las únicas opciones que tenés, se te va a hacer más pesado.

(Mujer, educación permanente, sector medio).

Sin embargo, estas elecciones, en los sectores sociales medios, se encuentran atravesadas por la tensión entre estudiar algo que «me gusta» y estudiar «algo con salida laboral». Cabe recordar que, estos sectores han recurrido a la educación (fundamentalmente a la pública) como recurso de movilidad social (Bocchio, y Miranda, 2018). No obstante, la creciente segmentación del sistema universitario y la devaluación de los títulos universitarios han producido una dificultad cada vez mayor para elegir una carrera terciaria o universitaria (Miranda, 2015). Las/os jóvenes dan cuenta de que sus prefiguraciones ocupacionales se ven condicionadas por las ideas que circulan en torno a la «salida laboral» de las carreras.

\section{P1: Yo creo que es diferente, hoy en día muchos eligen por conveniencia, por salida laboral, además te llenan mucho la cabeza, te tiran comentarios negativos de todo. P3: Todo muy lindo con el contenido pero cuando tengas que poner la olla...}

(Mujeres, educación permanente, sector medio). 


\subsection{Las elecciones en los sectores empobrecidos}

En los sectores sociales más empobrecidos de Argentina, terminar la escuela secundaria se configura como un verdadero logro. Actualmente -más de una década después de sancionada la obligatoriedad de la educación media- finalizar la escuela secundaria sigue siendo para muchas/os un desafío, a veces siendo las/os primeras/os de la familia en terminar este nivel educativo. En esta línea, coinciden con sus pares provenientes de sectores sociales medios en que cada vez es más necesaria la formación para acceder a un trabajo, pero es la escuela secundaria a la que apuntan como esencial para mejorar sus condiciones de vida. La subsistencia económica es un factor central en este grupo de jóvenes para decidir acerca de sus ocupaciones futuras. Por esa razón, la continuidad de los estudios superiores estaba, en la mayor parte de los casos, en duda. Entre quienes manifestaron la intención o el interés por continuar estudios superiores, hubo una marcada preferencia por las instituciones públicas de la ciudad, dado su carácter gratuito. Además, muchas/os trabajaban a la par que asistían a la escuela secundaria y manifestaban que seguirán haciéndolo al terminarla. Así, la continuidad de los estudios superiores estaba, en la mayoría de las ocasiones, pensada con actividad complementaria al trabajo. Asimismo, otra parte de las/os entrevistadas/os de este sector, manifestaron que estudiarían cursos relacionados a oficios ya que demandaban menor tiempo y costo. Se advierte, entonces, una jerarquización de las expectativas diferenciadas de acuerdo al sector social de donde provienen las/os jóvenes (Miranda, 2015), relacionada a qué estudiar, dónde y qué trabajos futuros piensan conseguir. Fundamentalmente, las/os jóvenes de sectores sociales desfavorecidos imaginan trabajos menos calificados que sus pares provenientes de sectores medios.

\section{P1: Más allá del «no querer estudiar», hay mucha gente que por su situación necesitan rápido tener un trabajo y no pueden estar mucho tiempo estudiando y tienen que trabajar y estudiar. Entonces, buscan carreras cortas para tener un título antes y un trabajo fijo más rápido para poder mantener a sus familias y esas cosas.}

(Mujer, educación permanente, sector bajo).

P3: Me inclino más por ir a trabajar, más que nada por la situación económica y el tiempo que toma un estudio, asi que, no lo veo como para mi estudiar.

P4: A mi me hubiera gustado también estudiar recursos humanos, yo me habia ido a anotar ya, pero por el tema económico preferí más hacer los cursos, que es lo que me gusta.

P1: O sea, cuando terminas el secundario tenés que trabajar, una ayuda tiene que llegar a tu casay estar estudiando te quita tiempo. Creo que podés hacer las dos cosas a la vez, pero te vas a estresar. (Varones, escuela educación permanente, sector bajo).

Se advierte, también, que el tiempo se configura como un recurso escaso para estas/os jóvenes, siendo un elemento en sí mismo que facilita o restringe las opciones. Por un lado, las/os entrevistadas/os mencionan que el tiempo que conllevan los estudios superiores en su totalidad dificulta «una salida laboral más rápida». Por otro lado, se menciona el tiempo que lleva estudiar en sí mismo además de trabajar, lo que implicaría una carga doble. Así, el tiempo es un recurso desigualmente distribuido, donde se juegan la autonomía y la coacción (Ramos Torre, 2007). 
A la vez, encontramos en expresiones como «no lo veo como para mi estudiar» o «no tengo la habilidad para ir a la universidad», una construcción de los estudios superiores como «imposibles». Como afirman Bourdieu y Paseron, (2009), esas construcciones condicionan las expectativas subjetivas y acaban por convertirse en determinantes de las vocaciones educativas a través de complejos procesos de violencia simbólica. De este modo, se naturalizan desigualdades entre los sectores socioeconómicos, que persisten en sus miradas hacia el futuro.

\subsection{Las mujeres de sectores empobrecidos}

La interseccionalidad como una perspectiva de análisis contempla relaciones sociales situadas, en la que los significados e importancia de distintas categorías dependen tanto del contexto como de los modos en que esas categorías son encarnadas por los sujetos para darles un significado (Platero, 2014). En ese sentido, las entrevistadas provenientes de sectores sociales más desfavorecidos mostraron cómo su posición de clase se imbrica complejamente con su condición de género para multiplicar los condicionamientos que experimentan en la vida cotidiana.

En primer lugar, la mayor parte de las madres de las/os jóvenes de sectores sociales desfavorecidos trabajaban fuera del hogar como niñeras o empleadas de casas particulares. Eran las que mayor carga doméstica tenían, compartiéndola principalmente con sus hijas. Muchas jóvenes de estos sectores eran las encargadas de cuidar a hermanas/os, sobrinas/os u otros familiares. El factor «tiempo» aparece nuevamente en las jóvenes de sectores sociales más desfavorecidos pero relacionado también a lo que Aguirre (2009) denomina «tiempo donado», es decir, el tiempo que se le dona a otra/o en razón de las características afectivo-morales de la relación establecida. Asimismo, los trabajos actuales de estas jóvenes giraban en torno a la producción o servicio de alimentos, la limpieza de hogares o el cuidado de niñas/os. Así, el tiempo «donado» sumado al tiempo que destinaban a sus actuales trabajos, limitaba los tiempos disponibles para estudiar.

\section{P3: Mi mamá está enferma, si le llega a pasar algo y yo me fui (...) También mis hermanos son chiquitos y la más grande no le da bola a mi vieja, sé que no la va a estar ayudando tanto como si yo estuviera acá con ella.}

(Mujeres, educación permanente, sector bajo).

En segundo lugar, sus ocupaciones prefiguradas se veían restringidas por su situación socio-económica, ya que debían elegir estudios que fueran gratuitos o trabajar en simultáneo para pagárselos. Pero además, su condición de mujeres las desalentaba de elegir determinadas ocupaciones, aun cuando éstas no representaran un costo económico ${ }^{3}$. Tal como advierte Bourdieu (2000), diversos mecanismos de violencia simbólica llevan a muchas mujeres a optar por salirse de lugares de poder y de alta competitividad, pero para aquellas que no se autoexcluyan o desarrollen «vocaciones no femeninas» vendrá la sanción social.

P2: En mi familia quieren que siga estudiando (...) pero ya cuando dije que quería estudiar en la facultad militar, mi abuelo dijo que no, «vos no porque sos una chica y las chicas no hacen eso».

\footnotetext{
3 Asistir a la escuela militar o a la escuela de policías suele ser una «salida laboral» frecuente para jóvenes de sectores desfavorecidos dado que ambas son gratuitas (deben rendir un examen de ingreso) y les permite estar en condiciones de sostenerse económicamente de manera rápida si logran superar las pruebas correspondientes.
} 


\title{
P1: A mi me pasó que con mi papa por ejemplo, a mi primero me gustaba policía, y después se me fue el gusto. \\ P2: A mi me bajonean un montón con eso. Te dicen tantas cosas y te desilusionan.
}

(Mujeres, educación permanente, sector bajo).

Finalmente, la maternidad (real o potencial) también emergió en estas jóvenes como una limitación a la hora de conseguir trabajo. Al consultarles acerca de sus expectativas en torno a la maternidad/ paternidad, tanto las/os jóvenes provenientes de sectores sociales medios como de sectores sociales bajos manifestaron deseos de tener hijos/as luego de haber alcanzado ciertas condiciones: la estabilidad económica y la estabilidad afectiva. Por otra parte, la idea de familia/pareja estable aparece en los relatos de los varones como condición necesaria para tener hijos/as, mientras que en las mujeres esa idea se discute y pierde centralidad como componente primordial. No obstante, ser «madres solas» adquirió connotaciones muy diferentes según el sector social. Mientras que para las mujeres de sectores sociales medios, tener y cuidar de sus hijas/os era algo «posible» si ocurría mientras estaban cursando sus estudios superiores, para las mujeres provenientes de sectores sociales más desfavorecidos, la maternidad se configuró como una amenaza de quedar excluidas, tanto de los estudios como del trabajo.

\author{
P1: En mi familia mi hermana llegó hasta quinto año pero como quedó embarazada no siguió \\ más. Y abora yo soy la que está en quinto y quiero tener un futuro. \\ M: ¿Creen que es posible hacer las dos cosas al mismo tiempo [tener hijas/os y estudiar]? \\ P4: Si tenés una persona que te acompaña y te ayuda, sí. \\ P5: Pero si estás sola no creo que se pueda. \\ (Mujeres, educación permanente, sector bajo).
}

\section{P2: Hay veces también que vas a un trabajo y te preguntan si tenés hijos o no y si decís que si ya no te dan el trabajo. A mi hermana se lo hicieron muchas veces. \\ M: ¿Y les parece que eso se lo preguntan a las mujeres y a los varones? \\ P3: Se lo preguntan más a las mujeres, porque bueno si el varón está buscando trabajo, «el hijo se queda con la madre» y es así. Cuando yo voy, no piensan que el bijo se va a quedar con el padre. \\ (Mujeres, escuela educación permanente, sector bajo).}

De este modo, se evidencia que para las muchas jóvenes, ser madres y contar con pocos recursos económicos, equivale a quedarse «sin futuro». Así, el estudio del trabajo doméstico desde una perspectiva interseccional es central para evidenciar desigualdades de género y de clase. En este sentido, el enfoque de la economía feminista, particularmente en América Latina, reconoce que las diferencias de género no existen en el vacío y que tanto mujeres como varones atraviesan las desigualdades estructurales (clase, etnia) de manera desigual. Por ello, el trabajo doméstico debe problematizarse y resolverse como un problema público dado que «las cadenas de valor de las mujeres son un eslabón fundamental para sostener la reproducción de la sociedad en que vivimos» (D’alessandro, 2019:65).

\section{Conclusiones}

A partir del análisis de las entrevistas, fue posible reconstruir algunos imaginarios, expectativas y deseos de jóvenes residentes en la ciudad de Rafaela, próximas/os a egresar de la escuela media. Ubicamos allí, 
modos de interiorizar expectativas y mandatos sociales, así como aspiraciones y preferencias que construyen sus subjetividades. El género y el nivel socio-económico emergieron como las dos variables de mayor impacto en esas elecciones, mientras que la modalidad de la escuela a la que asistían ocupó un lugar menos relevante, aunque mostró ciertas prácticas docentes que reforzaban la segregación en función del género.

En relación al género, encontramos que las/os entrevistadas/os prefiguran sus ocupaciones reproduciendo diversos estereotipos y mandatos. La preeminencia del modelo patriarcal clasifica y jerarquiza las ocupaciones, destinando a las mujeres a realizar trabajos y estudios peor remunerados y de menor prestigio social. En los casos estudiados fueron ocupaciones relacionadas a las ciencias humanas, el ámbito de la docencia y otras relacionadas a la rama de los «cuidados». Además, se evidenciaron diversos mecanismos de condicionamiento a esas elecciones que no sólo se relacionaban con ámbitos «habilitados» en función del género, sino también en función del trabajo doméstico y de cuidados que estaban mayoritariamente a cargo de las mujeres. A la vez, los relatos de las entrevistadas mostraron una construcción respecto del ser mujer como sujetos frágiles física y emocionalmente, lo que implica una supuesta necesidad de ser protegidas. Esto se relaciona íntimamente con la percepción de amenaza que se construye en relación al tránsito por el espacio público. Así, el miedo, como experiencia insistente en los relatos de las mujeres, está ausente en los relatos de los varones.

Asimismo, encontramos que algunas prácticas docentes reproducían las normativas tradicionales en función del género. Este hallazgo nos lleva a reflexionar acerca de la difícil implementación que ha tenido la Ley de Educación Sexual Integral sancionada en 2006, que apunta a la incorporación de la perspectiva de género en los planes de estudios de las escuelas. En este sentido, consideramos relevante que se continúen las investigaciones que abordan la efectiva aplicación de dicha ley en los establecimientos escolares y también otras acerca de la composición del estudiantado en los estudios superiores en función del género, ya que si bien se constata una «feminización» de la matrícula universitaria, esto aún no ha impactado fuertemente en la segregación horizontal y vertical del mercado laboral.

Por otra parte, la variable nivel socio-económico también atravesó las prefiguraciones acerca de futuras ocupaciones por parte de las/os jóvenes, evidenciando la persistencia de marcadas inequidades sociales. Por una parte, los sectores medios consideraron a los estudios superiores como indispensables para conseguir mejores condiciones laborales. Esta idea se vio tensionada por dos condiciones: disfrutar de los estudios/trabajos a futuro y tener «salida laboral». Sin embargo, en ninguno de los grupos provenientes de sectores medios se manifestaron preocupaciones respecto a cómo sostenerse económicamente mientras estudiaban ni con tener que pagar sus estudios en los casos en lo que eligieran instituciones privadas.

Por su parte, los sectores sociales más desfavorecidos manifestaron la necesidad imperiosa de terminar el secundario para conseguir una mejor inserción laboral. Muchas/os de ellas/os se encontraban trabajando a la par que estudiaban en la escuela secundaria. Por tanto, sus prefiguraciones respecto al futuro sostenían, la mayor parte de las veces, la idea de trabajar y estudiar a la vez. Asimismo, esas ocupaciones imaginadas, en términos generales, implicaban menor calificación que las proyectadas por los sectores medios y no ponderaban necesariamente el factor de disfrute en el trabajo que se realizaba. En ese sentido, varias/os de ellas/os pensaban continuar estudiando cursos de oficios que ofrecieran salidas laborales «mejores» y más «rápidas». Así, el factor «tiempo» cobró una dimensión fundamental para comprender esos relatos ya que condiciona las posibilidades en función del nivel socio-económico. Además, los estudios superiores se presentaron para algunas/os entrevistadas/os como «imposibles», mostrando 
el condicionamiento de las expectativas subjetivas que se produce en los sectores más desfavorecidos conduciendo a una «autoexclusión» en términos de Bourdieu y Paseron (2009).

Finalmente, encontramos que el grupo más vulnerado lo constituían las mujeres provenientes de sectores sociales empobrecidos, en donde la variable de género se imbricaba complejamente con su posición de clase. Desde una perspectiva interseccional, encontramos que el factor «tiempo» se configura como un condicionante más profundo para estas jóvenes dado que tenían a su cargo la mayor parte del trabajo doméstico y trabajaban a la par que cursaban la escuela. A la vez, enfrentaban todas las restricciones propias la segregación horizontal en el mundo del trabajo y los estudios superiores. Se evidenciaron diversos mecanismos de violencia simbólica que las llevaban a elegir ocupaciones «feminizadas» al tiempo que, aquellas que desarrollaban «vocaciones no femeninas» enfrentaba la sanción social de sus familiares. Estas restricciones se combinaron también con la maternidad (real o potencial) como factor que las excluye del mundo laboral, en donde ser joven, madre y contar con pocos recursos económicos equivale a quedarse «sin futuro». De este modo, la responsabilidad y el tiempo que consume el trabajo reproductivo en la vida cotidiana de las mujeres se constituyó como un componente clave para comprender que la procedencia de una determinada clase social condiciona el acceso y uso de recursos materiales, a la vez que da origen a otros tipos de desigualdades de género.

Así, las tendencias observadas marcan la persistencia de estereotipos, roles, permisos y limitaciones en función del género y del nivel socioeconómico, mas allá de la puesta en agenda de la perspectiva de género en la discursividad social y las políticas públicas argentinas en los últimos años. En ese sentido, género y clase social constituyen dimensiones complementarias del proceso de estratificación social general, por lo que deben analizarse considerando cómo el cruce entre ambas profundiza o disminuye la magnitud de la inequidad. Por ello, consideramos que la profundización de las líneas de investigación que abordan la «feminización de la pobreza» son centrales para dar cuenta de esos fenómenos.

Finalmente, consideramos que esta investigación realiza un aporte al conocimiento y la comprensión de los imaginarios juveniles y pone de manifiesto la percepción que estas/os jóvenes sostienen acerca de las estratificaciones de los mercados educativo y de trabajo. Profundizar en el estudio de los obstáculos, mandatos sociales, discriminaciones y vulneraciones que experimentan las/os jóvenes resulta fundamental para comprender las desigualdades persistentes, a la vez que permite interrogarse sobre su reproducción, tanto en términos institucionales como estructurales.

\section{Referencias bibliográficas}

Aguirre, Rosario (2009): "Uso del tiempo y desigualdades de género en el trabajo no remunerado" en Rosario Aguirre (cood.): Las bases invisibles del bienestar social. El trabajo no remunerado en Uruguay. Montevideo: Unifem.

Añover Lopez, Maria (2012): “Los espacios ‘del miedo', ciudad y género. Experiencias y percepciones en Zaragoza”. Geographicalia, 61, 25-45.

Arango Gaviria, Luz Gabriela (2006). Jóvenes en la universidad. Género, clase e identidad. Colombia: Siglo del Hombre Editores.

Baeza Leiva, Monica (2015): "Breve análisis de la feminización de la pobreza en Chile". Revista Chilena de Derecho y Ciencia Politica, 95-116. DOI: https://dx.doi.org/10.7770/rchdcp.v6i2.930. 
Bendit, René; Miranda, Ana (2013): “Trayectorias y expectativas de los egresados de la secundaria en Argentina". Documentos y aportes en administración pública y gestión estatal, 21 (13), 93-123. DOI: https://doi. org/10.14409/da.v1i21.4150.

Bocchio, Maria Cecilia; Miranda, Estela María (2018): "La escolaridad secundaria obligatoria en Argentina: Políticas para la inclusión social y educativa en la escuela". Revista Educación, 42 (2), 1-16. DOI: https://doi.org/10.15517/revedu.v42i2.27103.

Bourdieu, Pierre (2000). La dominación masculina. Barcelona: Editorial Anagrama.

Bourdieu, Pierre (2001). Poder, derecho y clases sociales. Bilbao: Desclée de Brouwer.

Bourdieu, Pierre; Paseron, Jean Claude (2009). Los herederos Los estudiantes y la cultura. Buenos Aires: Siglo XXI.

Butler, Judith (2001): El género en disputa: El feminismo y la subversión de la identidad. Mexico D.F.: Paidós.

Calderon, Yannet Paz; Campos Rios, Guillermo (2013): “Las mujeres jóvenes en méxico: ¿estudian o trabajan?”. Ultima Década, 63-83. DOI: http://dx.doi.org/10.4067/S0718-22362013000200004.

Cardini, Alejandra; D’Alessandre, Vanesa (2019). Transformar la educación secundaria. Buenos Aires: CIPPEC.

Casal Bataller, Joaquim (2000): “Capitalismo informacional, trayectorias sociales de los jóvenes y políticas de juventud” en Lorenzo Cachon Rodríguez (coord.): Jwventudes y Empleos: perspectivas comparadas. Madrid: INJUVE.

Castel, Robert (1997). La metamorfosis de la cuestión social: una cronica del salariado. Buenos Aires: Paidós.

Corica, Agustina (2012). Lo posible y lo deseable. Expectativas laborales de los jóvenes de la escuela secundaria. Buenos Aires: Fundación UOCRA. Editorial Aulas y Andamios.

Crenshaw, Kimberlé (2012): “Cartografiando los márgenes: Interseccionalidad, políticas identitarias y violencia contra las mujeres de color" en Raquel Lucas Platero (ed.): Intersecciones: cuerpos y sexualidades en la encrucijada. Barcelona: Belaterra.

D’Alessandro, Mercedes (2019). Economía feminista. Las mujeres, el trabajo y el amor. Buenos Aires: Sudamericana.

Duschastzky, Silvia y Corea, Cristina (2000). Chicos en banda, los caminos de la subjetividad en el declive de las instituciones. Buenos Aires: Paidós.

Esquivel, Valeria (2016): “La economía feminista en América Látina”. Nueva Sociedad, 265, 103-116.

EURYDICE (2009): Diferencia de género en los resultados educativos: medidas adoptadas y situación actual en Europa (en línea). DOI: http://doi.org/10.2797/48598, consultado el 10 de mayo de 2020.

Falu, Ana (2014): "El derecho de la mujeres a la ciudad. Espacios públicos sin discriminaciones y violencias". Revista Vivienda y Ciudad, 1, 10-28.

Filmus, Daniel (2015): “La universalización del secundario en America Látina” en Ana Miranda (cood.): Sociología de la educación y transición al mundo del trabajo. Buenos Aires: Teseo. 
Filmus, Daniel; Kaplan, Carina; Miranda, Ana y Moragues, Marina (2001). Cada vez más necesaria. cada vez. más insuficiente, la escuela media en épocas de globalización. Buenos Aires: Santillana.

Fraser, Nancy (1995): “¿De la redistribución al reconocimiento? Dilemas en torno a la justicia en una época 'postsocialista"” en Nancy Fraser: Iustitia Interrupta: Reflexiones críticas desde la posición "postsocialista". Santa Fe de Bogota: Siglo de Hombres Editores.

García Ramírez, Carmen Teresa (2016): “Contribuciones de la Teoría Social Feminista y los Estudios de Género a la sociología Venezolana”. Revista Espacio Abierto: cuaderno venezolano de socilogía, 25 (4), 47-60.

González Lopez, Isabel (2009): “La orientación académica y profesional en clave de igualdad”. Revista Participación educativa, 11, 110-121.

Guevara, Hilda y Belleli, Sandra (2015): "Las trayectorias académicas: dimensiones personales de una trayectoria estudiantil. Testimonio de un actor". Revista de Ciencias Sociales y Humanas, 4 (4),45-56.

Hochschild, Arlie (1983). The Managed Heart: The Commercialization of Human Feelings. Berkeley: California University Press.

Lagarde, Marcela (2006): "Por la vida y la libertad de las mujeres. Fin al feminicidio" en Diana Russell y Roberta Harmes (eds.): Feminicidio: una perspectiva global, México: Universidad Nacional Autónoma de México, Centro de Investigaciones Interdisciplinarias en Ciencias y Humanidades.

Machado País, José (2004): "Los bailes de la memoria: cuando el futuro es incierto". Revista JOVENes, $8(20), 74-95$.

Margulis, Mario (1996). La juventud es más que una palabra: ensayos sobre cultura y juventud. Buenos Aires: Paidós.

Miranda, Ana (2007). La nueva condición joven: educación, desigualdad y empleo. Buenos Aires: Fundación Octubre.

Miranda, Ana (2015). Sociología de la educación y la transición al mundo del trabajo. Buenos Aires: Teseo.

Miranda, Estela María (2013): "De la selección a la universalización. Los desafíos de la obligatoriedad de la educación secundaria”. Espacios en Blanco. Revista de Educación, 9-29.

Panaia, Marta (2013). Abandonar la Universidad con o sin título. Buenos Aires-Madrid: Miño y Dávila.

Lucas Platero, Raquel (2014): “Metáforas y articulaciones para una pedagogía crítica sobre la interseccionalidad”. Quaderns de Psicologia, 1 (16), 55-72. DOI: https://doi.org/10.5565/rev/qpsicologia.1219.

Pozzio, María (2012): "Análisis de género y estudios sobre profesiones: propuestas y desafíos de un diálogo posible -y alentador-”. Sudámerica: Revista de ciencias sociales, 1-31.

Ramos Torre, Ramón (2009): "Metáforas del tiempo en la vida cotidiana: una aproximación sociológica". Acta Sociologica, 49, 51-69. DOI: http://dx.doi.org/10.22201/fcpys.24484938e.2009.49.18704.

Roberts, Kenneth (1997): "Prolongued Transitions to Uncertain Destinations: The Implications for Careers Guidance". British Journal of Guidance an Couselling, 25, 345-360. DOI: https://doi. org/10.1080/03069889708253813. 
Scott, Joan (1999). Gender and the Politics of History. Nueva York: Universidad de Columbia.

Senett, Richard (2000). La corrosión del carácter. Las consecuencias personales del trabajo en el nuevo capitalismo. Barcelona: Anagrama.

Subirats, Marina (1999): “Género y escuela” en Carlos Lomas (coord.): ¿Iguales o diferentes? Género, diferencia sexual, lenguaje y educación. Buenos Aires: Paidós.

Subirats, Marina y Brullet, Cristina (1988). Rosa y azul. La transmisión de los géneros en la escuela mixta. Madrid: Instituto de la Mujer.

White Robert Douglas y Wyn, Johanna (2008). Youth and Society: Exploring the Social Dynamics of Youth Experience. Melbourne: Oxford University Press.

\section{Notas biográficas}

Cecilia Dionisio es Licenciada en Psicología y Especialista en Psicología Clínica de la Universidad Nacional de Córdoba (Argentina). Es Docente del Departamento de Cultura, Educación y Conocimiento de la Universidad Nacional de Rafaela (Argentina) donde también integra el Grupo de Estudio e Investigación en Teorías de Género, Diversidad e Interseccionalidad. Participa de proyectos de investigación relacionados a juventudes, educación e inclusión al mundo del trabajo desde la perspectiva de género. Además, es doctoranda del Programa de Doctorado en Ciencias Sociales de la Universidad de Buenos Aires (Argentina) y coordina el Programa de Fortalecimiento al Ingreso y la Permanencia de las/os estudiantes de la Universidad Nacional de Rafaela.

Hugo Lewin es Sociólogo y Doctor en Ciencias Sociales por la Universidad de Buenos Aires (Argentina). Es Profesor Asociado de Cultura y Subjetividad en la Universidad Nacional de Rafaela (Argentina) y Profesor Adjunto de Sociología de la Cultura en la Universidad de Buenos Aires, donde además es miembro del Instituto de Investigaciones Gino Germani. Allí integra desde hace treinta años un equipo dedicado a la investigación de la cultura en sus múltiples articulaciones con distintos fenómenos sociales: la vida urbana, la discriminación social, la sexualidad, la juventud, los procesos de informatización y digitalización de la vida, entre otras problemáticas. En la Universidad Nacional de Rafaela dirige una investigación enfocada en los imaginarios ocupacionales juveniles y la vinculación de éstos con variables sociológicas clásicas: nivel socio-económico, género y modalidades educativas. Actualmente dirige la Carrera de Sociología de la UBA. 